\title{
Spina Bifida-Ultrasonographic Diagnosis in First and Second Trimesters
}

\author{
${ }^{1}$ Laura Sarno, ${ }^{2}$ Gabriele Saccone, ${ }^{3}$ Marco Di Cresce, ${ }^{4}$ Pasquale Martinelli, ${ }^{5}$ Giuseppe Maria Maruotti
}

\begin{abstract}
Accurate and timely prenatal diagnosis of spina bifida (SB) is a major challenge of actual antenatal care. The diagnosis of spina bifida may be only suspected during I trimester because the detection rate of intracranial traslucency is $50 \%$; the final diagnosis is made in the II trimester by direct visualization of defect of spine or the presence of myelomeningocele or the visualization of indirect signs. When a spine defect is detected it is necessary a neurosurgical counseling with the patient. The degree of handicap and the survival rate depend on the level of injury, the size of the defect, and the presence of associated anomalies. In tertiary fetal medicine centers, two-dimensional (2D) and three-dimensional (3D) ultrasound allows an accurate determination of the location, type, extention of the defect. Maternal-fetal surgery for myelomeningocele repair must be offered to carefully selected patients even if there are significant maternal implications and complications
\end{abstract}

Keywords: Intracranial traslucency, Myelomeningocele, Prenatal diagnosis, Spina bifida, Ultrasonography I and II Trimester

How to cite this article: Sarno L, Saccone G, Di Cresce M, Martinelli P, Maruotti GM. Spina Bifida-Ultrasonographic Diagnosis in First and Second Trimesters. Donald School J Ultrasound Obstet Gynecol 2017;11(4):341-346.

Source of support: Nil

Conflict of interest: None

\section{INTRODUCTION}

Neural tube defects (NTD) result from an alterated neurulation in the third or fourth week of development and thus represent the earliest manifestation of organ malformation. Neural tube defects are classified into cranial dysraphism leading to anencephaly or meningoencephalocele and spinal dysraphism with or without meningomyelocele. The prevalence of NTD worldwide is 1 to 2 per 1,000 neonates. Myelomeningocele, a severe form of spina bifida (SB), occurs in approximately 1 in 3,000 live births. The incidence of NTDs in autopsied fetuses with malformations was $10 \%$. Analysis of the sex

\footnotetext{
1-3,5 Medical Doctor, ${ }^{4}$ Professor

${ }^{1-5}$ Prenatal Diagnosis Unit, Department of Neuroscience Reproductive Sciences and Dentistry, School of Medicine University of Naples, Naples, Italy
}

Corresponding Author: Laura Sarno, Medical Doctor, Prenatal Diagnosis Unit, Department of Neuroscience, Reproductive Sciences and Dentistry, School of Medicine, University of Naples, Naples, Italy, e-mail: laurettasarno@gmail.com distribution showed a female preponderance in cranial dysraphisms, but the sex distribution of SB cases was equal. The localization and extent of NTDs varied, with lumbosacral cases clearly predominating. The proportion of isolated, associated, and syndromic NTD was 56, 23.5, and $20.6 \%$ respectively. In the majority of syndromes, the NTD represented a not previously observed syndromic feature. ${ }^{1}$

\section{SPINA BIFIDA}

Spina bifida is the most common malformation of the central nervous system. It could be defined as a vertebral median defect, with external exposure of the spinal cord. In most cases, the defect could be found in the dorsal part of the vertebral arch, while the splitting of the vertebral body represents only rarely the defect. Its incidence changes according to several factors: Geographical area, ethnic differences, and seasonal variations; it is more common in Caucasians than Eastern, and such a difference persists after migrations, suggesting therefore, a genetic background more than an environmental one. Its incidence shows a 20-fold increase in diabetics, a 10- to 20 -fold increase in patients taking valproic acid during their first trimester of pregnancy, a 30-fold increase in case of a previous affected son, and a 60-fold increase with two sons affected. ${ }^{2}$ The lesions characterizing the SB could be distinguished in ventral and dorsal lesions. Ventral lesions are extremely rare, and characterized by the splitting of the vertebral body; such lesions are usually located at the lower cervical or upper thoracic part of the spine.

Dorsal defects are more common and classified as: closed SB and open SB.

Closed SB represents about $15 \%$ of cases, and it consists of a small defect completely covered by normal skin. Such condition is often asymptomatic and is diagnosed incidentally or after a radiological scan of the column. In other cases, hypertrichosis or a pigmented area, or a subcutaneous lipoma, could be found over the defect. The clinical relevance of this lesion is its frequent association with spinal cord infections.

The open SB, instead, is the most common lesion and it is found in $85 \%$ of the cases. The spinal cord may be exposed or the defect can be covered by the meningeal membranes. The lesion often looks like a cyst: if only the 


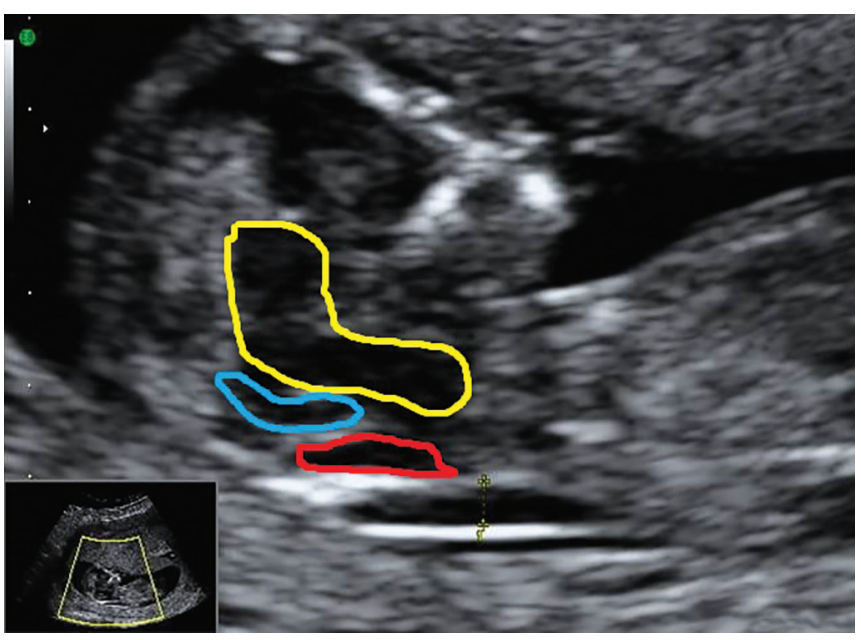

Fig. 1: Sagittal scan used for screening in the first trimester. It is possible to evaluate the anteroposterior diameter of the fourth ventricle (inner translucency) corresponding to an anechogenic space (blue space), between two hyperechogenic lines, the posterior margin of the diencephalus anteriorly (yellow space), and the choroidal plexus of the fourth ventricle (red space)

meninges are included in the cystic lesion, it is referred to as meningocele, if there are meninges and spinal cord inside, the word myelomeningocele has to be used. ${ }^{3}$

\section{Ultrasonographic Diagnosis in the First Trimester}

In the last years, several markers have been proposed for the diagnosis of SB in the 11 to 14 weeks scan. The first marker introduced was the intracranial translucency, i.e., an anechoic space between two hyperechoic lines (the posterior margin of the diencephalus anteriorly and the choroidal plexus of the fourth ventricle posteriorly; Fig. 1).

Chaoui and Nicolaides ${ }^{4}$ reported the absence of the intracranial translucency in four cases of spina open bifida during the first trimester scan. Later, the same authors find out, on a 30-case analysis, as a sign of gradual loss of liquor through the defect, a shift of the diencephalus toward the occipital bone, with the disappearance of the cisterna magna. In the first trimester, this turns into an inversion of brain stem:brain stem/occipital bone ratio (BS:BS/OB), which normally results to be $<1{ }^{5,6}$ The correct acquisition technique is explained in Figure 2.

In the axial plane, if two lines are traced along the margins of the cerebral peduncles and thalamus, they tend to form an acute angle in a normal fetus. In the open SB, instead, there is a posterior shift of the cerebral peduncles, so the two lines tend to become parallel. ${ }^{5}$

In the first trimester, it is possible to observe an anechoic space corresponding to the third ventricle and, more posteriorly, another anechoic space corresponding to the Sylvius aqueduct, well separated from the occipital bone; the lateral ventricles and their choroid

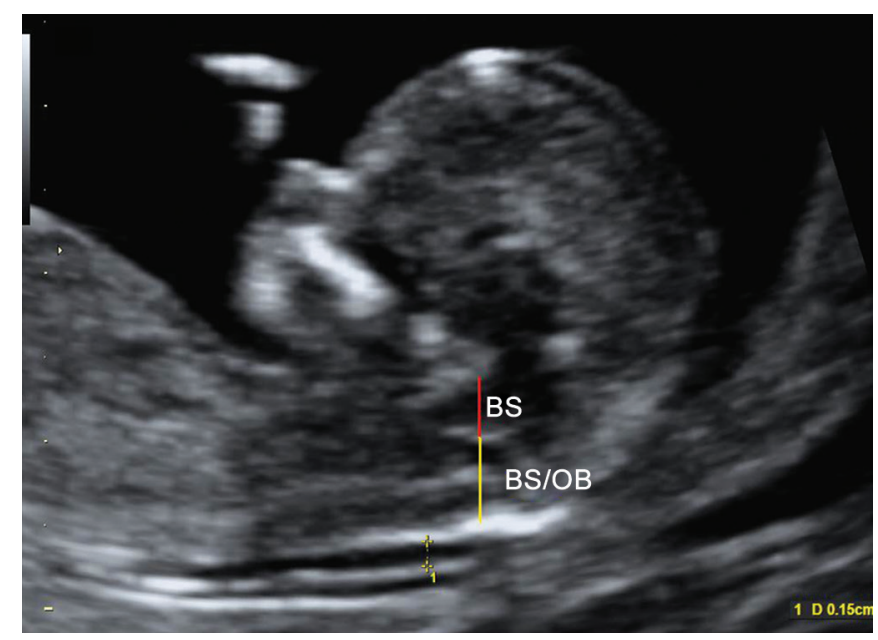

Fig. 2: Brain stem:brain stem/occipital bone ratio (BS:BS/OB). It normally results to be $<1$

plexi are well-evident too. The loss of liquor resulting from the spinal defect leads to a reduction in the ventricular spaces, which is already evident in the first trimester. In fetuses with open SB, in fact, a reduction in lateral ventricular diameters, third ventricle's roof, Sylvius aqueduct, and fourth ventricle can be found. ${ }^{7}$ As in the second trimester also in the first trimester a reduction in the biparietal diameter could be seen in fetuses with SB. ${ }^{7}$ In 2013, Mangione et $\mathrm{al}^{8}$ reported the sensitivity of several markers for the diagnosis of $\mathrm{SB}$ in the first trimester. Specifically, the nonvisualization of the cisterna magna was associated with a sensitivity of 50 to $73 \%$, while the lack of visualization of intracranial translucency was associated with a sensitivity of 29 to $48 \%$. Overall, any abnormality of the posterior fossa was associated with a sensitivity of 50 to $90 \%$. Likewise, a recent meta-analysis of 21,070 fetuses reported a modest sensitivity of intracranial translucency in the diagnosis of open SB (53\%), but a high specificity $(99.7 \%) .{ }^{9}$ Although there have been many efforts to improve the diagnosis of SB in the first trimester, most cases are still diagnosed later in the second trimester.

\section{Ultrasonographic Diagnosis in the Second Trimester}

Compared with anencephaly and encephalocele, commonly diagnosed earlier in pregnancy, the diagnosis of $\mathrm{SB}$ is in most cases performed during the second trimester scan, still considered as the principal screening of the NTD. The ultrasound diagnosis of SB in the second trimester is based on the detection of direct signs, i.e., the direct visualization of the spine defect, and of indirect signs, which can be identified by examining the fetal head. The study of the fetal spine, in the second trimester of pregnancy, should be performed through three planes: coronal, axial, and sagittal (Fig. 3). 


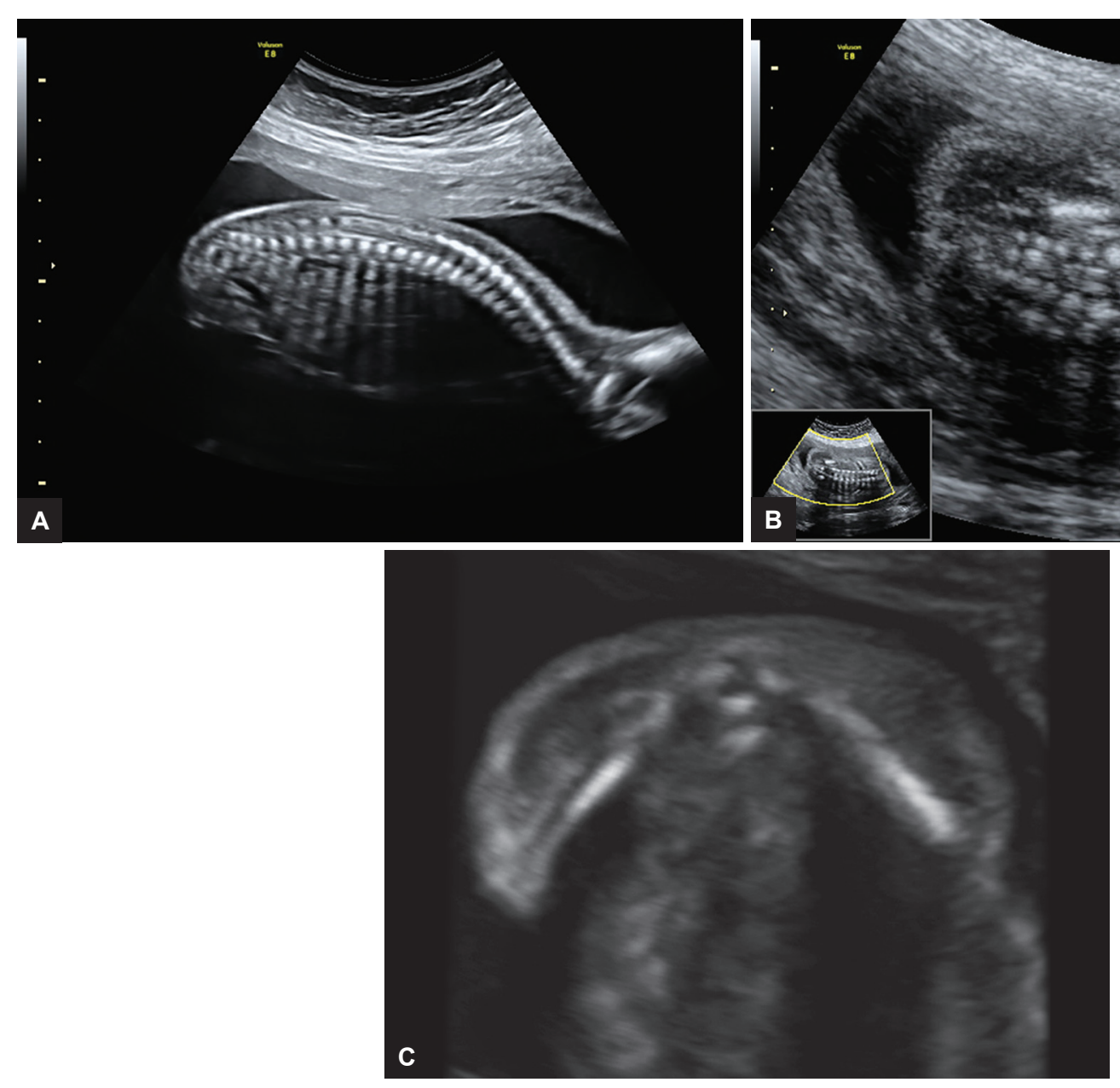

Figs 3A to C: (A) Sagittal view of spine (two parallel lines corresponding to the spinous vertebral processes and vertebral bodies converging into the sacrum, and the skin covering the spine); (B) coronal view of spine (three parallel lines); and (C) axial view of spine

The diagnosis of $\mathrm{SB}$ is made through both soft tissues and skeletal signs. The soft tissue markers are the absence of the skin and the subcutaneous tissues that face the defect and the presence of a protrusion that may correspond to a meningocele or myelomeningocele. Skeletal signs derive from the vertebral anomalies associated with SB.

The optimal knowledge of the spine anatomy scans is a basic requirement for the diagnosis. There are three major scan planes: the sagittal plane, the transverse plane, and the coronal plane. In the sagittal plane, the spine appears normally as two parallel lines converging into the sacrum. The two lines correspond to the processes spinosus and vertebral bodies. In cases of SB, the disappearance of the posterior line and the overlapping of the soft tissues are observed (Fig. 4).

It is essential to visualize the continuity of the skin above the column; in this scan, as well as in the axial one, you need to make sure there is amniotic fluid between the skin and the uterine wall, which would allow the visualization of the myelomeningocele. The sagittal scan is the one that allows us to measure the size of the defect and allows us to evaluate any curvatures that, if accentuated, are an indirect spinal vertebral sign. In the coronal

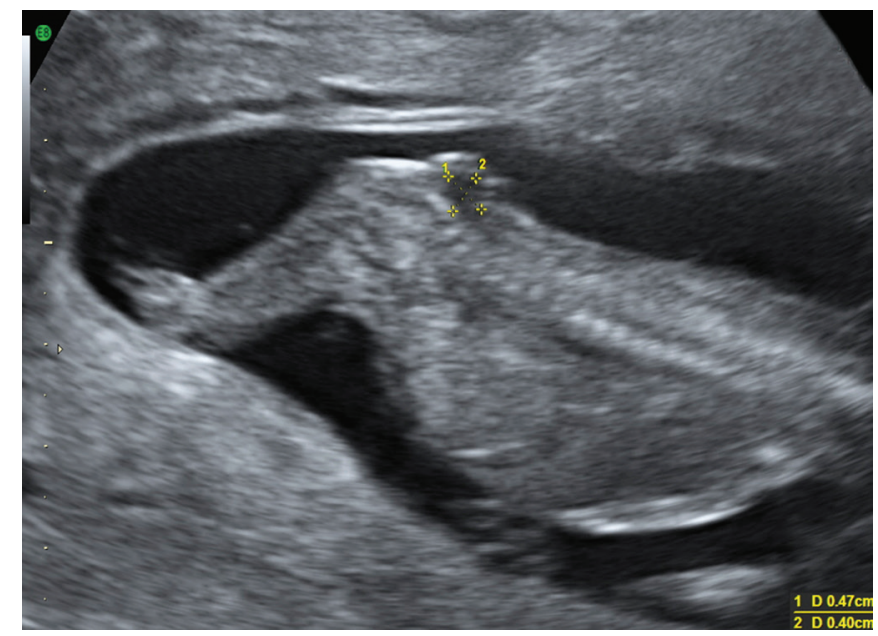

Fig. 4: Sagittal plane, the spine appears abnormal because we do not observe two parallel lines; there is the disappearance of the posterior line and the overlapping of the soft tissues

plane, the column appears as two or three parallel lines. The defect will appear as the enlargement of the typical image of the spine in the tract affected, due to the divergent separation of vertebral lateral processes. Sometimes, trying to see the overlying skin, an anecogenic minus corresponding myelomeningocele will be identified. 


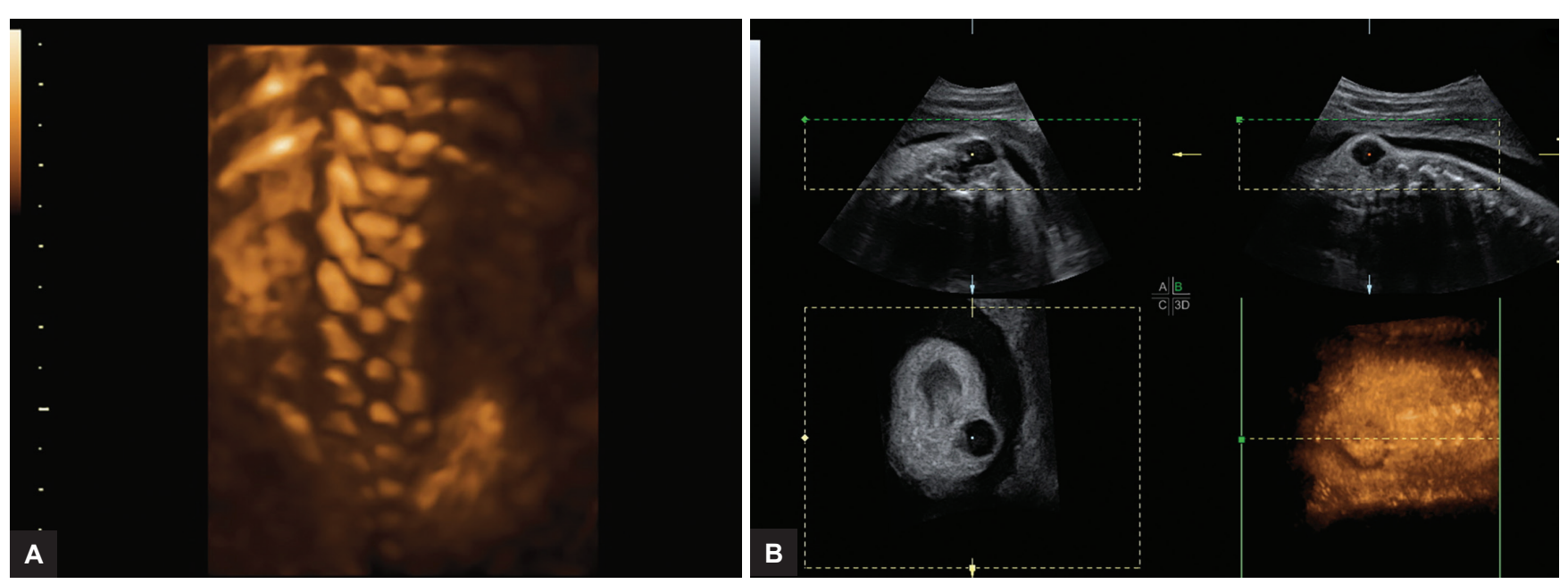

Figs 5A and B: (A) A 3D scan for the evaluation of the spine defect; and (B) it is possible the perfect reconstruction of the small defect (green arrow)

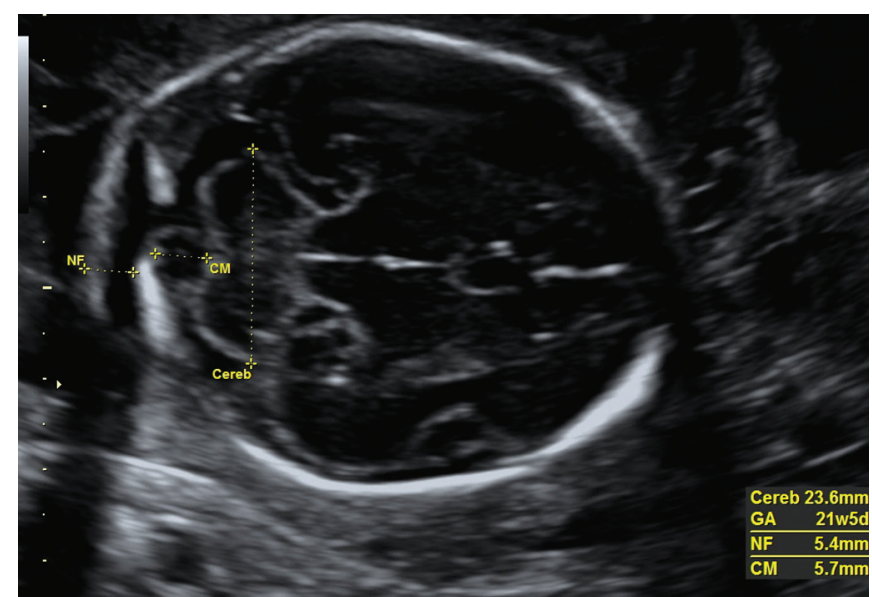

Fig. 6: A regular transcerebellar scan. This scan is used for the evaluation of the posterior fossa. The cisterna magna (CM) is not obliterated

In the axial plane, the vertebral canal appears as a closed circle; it is anteriorly bounded by the ossification center of the vertebral body and posteriorly by the two ossification centers of the laminae. In case of a defect, the posterior part is absent and the vertebra affected will be deformed with a "C" or " $\mathrm{U}$ " shape; it will also be possible to see the protrusion of the myelomeningocele. ${ }^{10}$ The skin and the muscles above the defect are absent. The ultrasound detection rate is $82 \%$. The $3 \mathrm{D}$ ultrasound plays an important role in the study of the spine. In the volume acquisition, the starting scan is in the sagittal plane, and a rotation angle of 30 to $40^{\circ}$ is sufficient in the majority of cases (Fig. 5).

The main advantage of 3D scanning is linked to the simultaneous viewing of the three vertebral processes of each vertebrae, and the possibility to rotate the volume according to the three axial planes, allowing easier detection of small defects. ${ }^{11-15}$ Acquiring a normal volume, however, does not exclude diagnosis of SB. ${ }^{16}$

Not always the defects are identified through the study of the spine alone; often the indirect signs at the

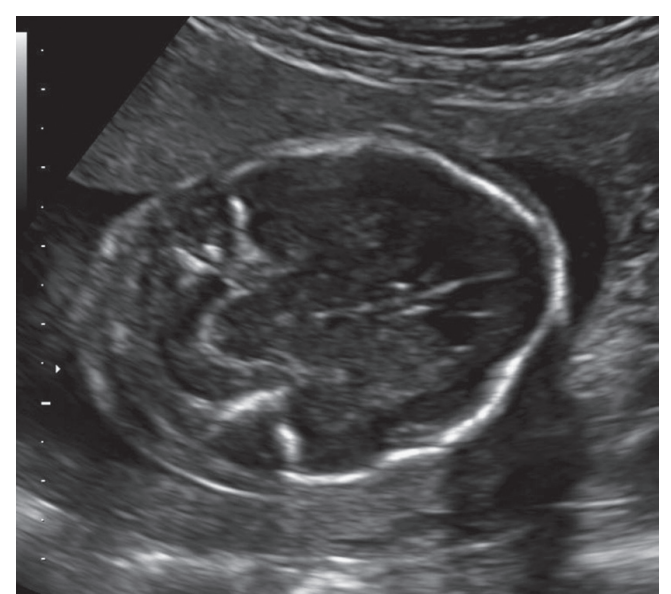

Fig. 7: A transcerebellar "banana sign." There is obliteration of the cisterna magna, and the cerebellum assumes a banana-like shape

level of the fetal brain guide us to the diagnosis. As mentioned above, the SB can be open (when there is a skin involvement and the neural canal is exposed and protrudes) or occult (when the vertebral defect is covered by skin). In open SB, the protrusion through the defect causes a caudal regression of the cerebellum and the cerebral trunk, with obliteration of the cisterna magna (Chiari II malformation). The most important scan is, therefore, the transcerebellar (Fig. 6) where it is possible to evaluate the posterior fossa.

The main indirect signs are

- Banana sign (Fig. 7): abnormalities of the cerebellar hemispheres, which assume a banana-like shape, with obliteration of the cisterna magna,;

- Lemon sign: deformation of the frontal bone, so that the skull assumes a lemon-like appearance, ${ }^{17}$

- Biparetal diameter and cranial circumference $<5$ th percentile, ${ }^{17,18}$

- Cerebellar diameter $<5$ th centile ${ }^{19}$

- Hydrocephaly: it is most likely caused by Chiari II malformation, associated with an obstructed flow of 


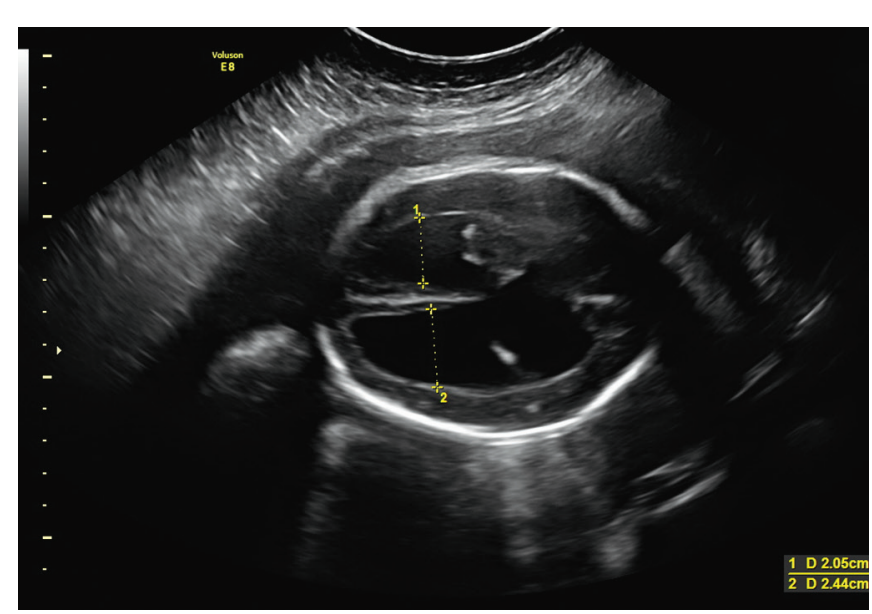

Fig. 8: Ventricular point. Typical shape of the ventricular horns was noted

the liquor through the fourth ventricle or with the stenosis of the Sylvius aqueduct that may be present in 40 to $75 \%$ of cases. $^{20}$

Recently, other indirect signs of SB have been identified. Among these, we remember the ventricular point (Fig. 8), which consists in a modification of the shape of the occipital horn that will appear pointed rather than circular. Callen and Filly ${ }^{21}$ found this sign in $70 \%$ of the examined fetuses.

\section{CONCLUSION}

When a spine defect is detected, it is necessary a neurosurgical counseling with the patient; the risk of recurrence is 2 to $5 \%$. The children with SB may have walking problems, sphincter dysfunction, sexual dysfunctions, skeletal deformities, cognitive disease.

The degree of handicap and the survival rate depend on the level of injury, the size of the defect, and the presence of associated anomalies. The lower the injury level, the better the prognosis. The cognitive outcome is related by the presence or absence of hydrocephaly. Altogether about $70 \%$ of children with SB will have IQ $>80$.

Accurate and timely prenatal diagnosis of SB is a major goal of modern antenatal care. The diagnosis of SB may be only suspected during I trimester because the detection rate of intracranial traslucency is $50 \%$; the final diagnosis is made in the II trimester by direct visualization of defect of spine or the presence of myelomeningocele or the visualization of indirect signs. In tertiary fetal medicine centers, 2D and 3D ultrasound allows an accurate determination of the location, type, extent, and upper level of the spinal defect as well as the presence of associated anomalies. Women with pregnancies complicated by fetal myelomeningocele who meet established criteria for in utero repair should be counseled in a nondirective fashion regarding all management options, including the possibility of open maternal-fetal surgery. Open maternal-fetal surgery for myelomeningocele repair is a major procedure for the woman and her affected fetus. There are significant maternal implications and complications that may occur acutely, postoperatively, for the duration of the pregnancy, and in subsequent pregnancies. Maternal-fetal surgery for myelomeningocele repair should be offered only to carefully selected patients.

\section{REFERENCES}

1. Schoner K, Axt-Fliedner R, Bald R, Fritz B, Kohlhase J, Kohl T, Rehder H. Fetal pathology of neural tube defects-an overview of 68 cases. Geburtshilfe Frauenheilkd 2017 May;77(5):495-507.

2. Main DM, Mennuti MT. Neural tube defects: issues in prenatal diagnosis and counselling. Obstet Gynecol 1986 Jan;67(1):1-16.

3. Romero R, Pilu G, Jeanty P, Ghidini A, Hobbins JC. Prenatal diagnosis of congenital anomalies. Neural tube defects. East Norwalk (CT): Appleton \& Lange; 1988.

4. Chaoui R, Nicolaides KH. From nuchal translucency to intracranial translucency: towards the early detection of spina bifida. Ultrasound Obstet Gynecol 2010 Feb;35(2):133-138.

5. Chaoui R, Nicolaides KH. Detecting open spina bifida at the 11-13-week scan by assessing intracranial translucency and the posterior brain region: mid-sagittal or axial plane? Ultrasound Obstet Gynecol 2011 Dec;38(6):609-612.

6. Scheier M, Lachmann R, Petros M, Nicolaides KH. Threedimensional sonography of the posterior fossa in fetuses with open spina bifida at 11-13 weeks' gestation. Ultrasound Obstet Gynecol 2011 Dec;38(6):625-629.

7. Loureiro T, Ushakov F, Montenegro N, Gielchinsky Y, Nicolaides KH. Cerebral ventricular system in fetuses with open spina bifida at 11-13 weeks' gestation. Ultrasound Obstet Gynecol 2012 Jun;39(6):620-624.

8. Mangione R, Dhombres F, Lelong N, Amat S, Atoub F, Friszer S, Khoshnood B, Jouannic JM. Screening for fetal spina bifida at 11-13 week scan using three anatomical features of the posterior brain. Ultrasound Obstet Gynecol 2013 Oct;42(4):416-420.

9. Maruotti GM, Saccone G, D'Antonio F, Berghella V, Sarno L, Morlando M, Giudicepietro A, Martinelli P. Diagnostic accuracy of intracranial translucency in detecting spina bifida: a systematic review and meta-analysis. Prenat Diagn 2016 Nov;36(11):991-996.

10. Paladini D, Volpe P. Diagnostica differenziale ed inquadramento delle anomalie del sistema nervoso. In: Anomalie congenite fetali: Diagnosi differenziale ed indicatori ecografia di prognosi. Di Canosa Editore; 2004.

11. Mueller GM, Weiner CP, Yankowitz J. Three-dimensional ultrasound in the evaluation of fetal head and spine anomalies. Obstet Gynecol 1996 Sep;88(3):372-378.

12. Johnson DD, Pretorius DH, Riccabona M, Budorick NE, Nelson TR. Three-dimensional ultrasound of the fetal spine. Obstet Gynecol 1997 Mar;89(3):434-438.

13. Riccabona M, Johnson D, Pretorius DH, Nelson TR. Three dimensional ultrasound: display modalities in the fetal spine and thorax. Eur J Radiol 1996 May;22(2):141-145.

14. Yanagihara $T$, Hata T. Three-dimensional sonographic visualization of fetal skeleton in the second trimester of pregnancy. Gynecol Obstet Invest 2000;49(1):12-16. 
15. Lee W, Chaiworapongsa T, Romero R, Williams R, McNie B, Johnson A, Treadwell M, Comstock CH. Adiagnostic approach for the evaluation of spina bifida by three-dimensional ultrasonography. J Ultrasound Med 2002 Jun;21(6):619-626.

16. Pilu G, Ghi T, Carletti A, Segata M, Perolo A, Rizzo N. Three dimensional ultrasound examination of the fetal central nervous system. Ultrasound Obstet Gynecol 2007 Aug;30(2):233-245.

17. Nicolaides KH, Campbell S, Gabbe SG, Guidetti R. Ultrasound screening for spina bifida: cranial and cerebellar signs. Lancet 1986 Jul 12; 2(8498):72-74

18. Cuppen I, de Bruijin D, Geerdink N, Rotteveel JJ, Willemsen MA, van Vugt JM, Pasman JW, Roeleveld N. Small biparietal diameter and head circumference are part of the phenotype instead of independent prognostic markers in fetuses with spinal dysraphism. Fetal Diagn Ther 2015;37(2):135-140.

19. Bahlmann F, Reinhard I, Shramm T, Geipel A, Gembruch U, von Kaisenberg CS, Schmitz R, Stupin J, Chaoui R, Karl K, et al. Cranial and cerebral signs in the diagnosis of spina bifida between 18 and 22 weeks of gestation: a German multi center study. Prenat Diagn 2015 Mar;35(3):228-235.

20. Carter CO, Evans KA, Till K. Spinal dysraphism: genetic relation to neural tube malformations. J Med Genet 1976 Oct;13(5):434-450.

21. Callen AL, Filly RA. Supratentorial abnormalities in the Chiari II malformation, I: the ventricular "point". J Ultrasound Med 2008 Jan;27(1):33-38. 